\title{
Manifestaciones oftalmológicas de la hipertensión arterial
}

\section{Ophthalmological manifestations of arterial hypertension}

\author{
N. A. Rodríguez, A. Zurutuza
}

\section{RESUMEN}

La hipertensión arterial sistémica (HTA) es una de las enfermedades más frecuentes en los países industrializados, llegando su incidencia hasta un $30 \%$, cifra que va en aumento por la mayor esperanza de vida de la población. Esta elevación de la presión arterial ocasiona o acelera los cambios en la pared vascular de los órganos diana como el riñón, cerebro, corazón y ojo.

A nivel ocular, la HTA produce lesiones en la retina, la coroides y cabeza del nervio óptico, pudiendo abarcar un amplio rango de lesiones, desde un estrechamiento vascular leve hasta una pérdida visual severa por neuropatía óptica isquémica.

La respuesta primaria de las arterias retinianas a la hipertensión arterial sistémica es un estrechamiento vascular y los signos que aparecen en el fondo de ojo en la retinopatía hipertensiva son la vasoconstricción difusa o focal, la extravasación por permeabilidad vascular aumentada y la arteriolosclerosis con engrosamiento de la pared de los vasos. Estas tres entidades son las responsables de la aparición de diferentes lesiones que van a caracterizar los estadíos de la enfermedad retiniana como son: los cruces arteriovenosos, exudados duros y algodonosos, trombosis, embolias, hemorragias en el parénquima retiniano, desprendimiento seroso de retina, edema de papila y neuropatía óptica isquémica en los casos más severos como el caso de la hipertensión arterial maligna.

Palabras clave. Hipertensión arterial. Retinopatía hipertensiva. Exudados retinianos. Cruces arteriovenosos.

An. Sist. Sanit. Navar. 2008; 31 (Supl. 3): 13-22.

\begin{abstract}
Systemic Arterial hypertension (AHT) is one of the most frequent diseases in the industrialised countries, with an incidence reaching $30 \%$, a figure that is rising due to the greater life expectancy of the population. This rise in arterial tension causes, or accelerates, changes in the vascular wall of the target organs such as the kidney, brain, heart and eye.

At the ocular level, AHT produces lesions in the retina, the choroids and optic nerve head; this can include a wide range of lesions, from slight vascular narrowing to severe visual loss due to ischaemic optical neuropathy.

The primary response of the retinal arteries to systemic arterial hypertension is vascular narrowing and the manifestations that appear at the back of the eye in hypertensive retinopathy are diffuse or focal vasoconstriction, extravasation due to increased vascular permeability and arteriosclerosis with swelling of the wall of the vessels. These three entities are responsible for the appearance of different lesions that characterise the stages of the retinal disease, which are: arteriovenous crossings, hard and cottonlike exudates, thrombosis, embolisms, haemorrhages in the retinal parenchyma, vitreous detachment from the retina, papilla edema and ischaemic optical neuropathy in the more severe cases, such as in case malign arterial hypertension.
\end{abstract}

Key words. Arterial hypertension. Hypertensive retinopathy. Retinal exudates. Arteriovenous crossings.
Servicio de Oftalmología. Hospital de Navarra. Pamplona.

\section{Correspondencia:}

Nelson Arturo Rodríguez Marco

Servicio de Oftalmología

Hospital de Navarra

Tfno. 848422194

Irunlarrea, 3

31008 Pamplona 


\section{INTRODUCCIÓN}

La hipertensión arterial (HTA) es una de las enfermedades más frecuentes en la población de los países industrializados, siendo su incidencia en los países desarrollados de hasta un 30\%. La importancia de esta enfermedad radica en los cambios producidos en la pared de arterias y venas, que son factores de riesgo que conducen a daños a nivel del riñón, corazón, cerebro y retina. En lo que se refiere a las lesiones a nivel ocular la HTA produce cambios en el parénquima retiniano, coroides y cabeza del nervio óptico. Las alteraciones que la HTA origina en los vasos y en la retina se producen a través de dos mecanismos:

1. Aumento del tono de las arteriolas que conduce a la vasoconstricción e hiperplasia de la capa muscular de las arteriolas.

2. Esclerosis reactiva por una acumulación de material hialino que hace a la arteriola más rígida y menos sensible a estímulos vasopresores.

La transparencia de los medios oculares permite un examen detallado de las alteraciones vasculares que nos informará de la repercusión sistémica de la enfermedad hipertensiva y arteriosclerosis, así como el seguimiento y pronóstico de la enfermedad ${ }^{1}$. El tratamiento de la retinopatía hipertensiva consiste en el control eficaz y mantenimiento de la presión arterial.

\section{FISIOPATOLOGÍA}

Las alteraciones de la HTA a nivel de los vasos retinianos se producen a través de dos vías:

\section{Vía de la hipertonía}

Da lugar a vasoconstricción, hiperplasia muscular y necrosis fibrinoide como respuesta automática y generalizada ante la HTA.

\section{Vía de la esclerosis}

Por acumulación de material hialino en la íntima y en la elástica interna que se extiende hacia la túnica media asociándose a atrofia de la capa muscular que es sustituida por material hialino en un proceso denominado arterioesclerosis con lo que la arteria se vuelve rígida dejando de ser contráctil $^{2}$ (Tabla 1).

\section{SIGNOS OFTALMOLÓGICOS DEL SÍNDROME VASCULAR HIPERTENSIVO}

\section{Cruce arteriovenoso}

Son consecuencia de la esclerosis arteriolar secundaria a la HTA crónica (esclerosis reactiva) o a la edad avanzada (esclerosis involutiva). La HTA mantenida en el tiempo produce un adelgazamiento de la capa media de la arteriola y proliferación del tejido perivascular que da lugar a un aumento de grosor y endurecimiento de la adventicia común entre arteria y vena, que producirá una constricción simultánea de la arteriola y la vénula con la consiguiente deformidad de la pared venosa ya que ésta tiene menos consistencia. Los signos clásicos de cruce arteriovenoso (AV) son $^{3,4}$ (Figs. 1, 2 y 3):

- Signo de Gunn: valora el calibre de la vénula y mide la deformidad de ésta a nivel del cruce AV que conlleva un éstasis vascular venoso distalmente al mismo. Hay cuatro grados:

- Grado I: ocultamiento de la vena por la arteria.

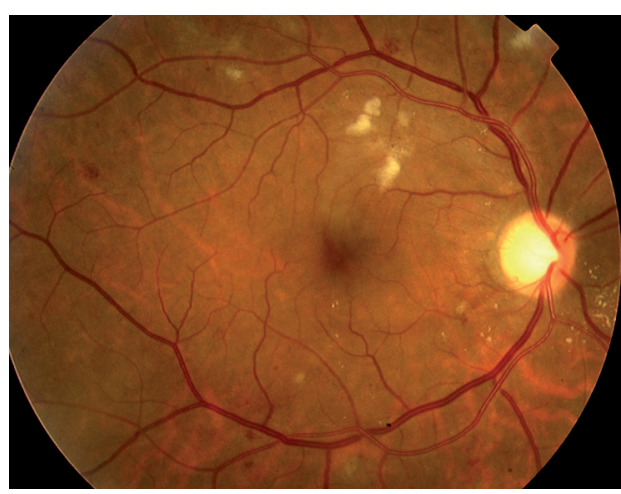

Figura 1. Imagen que muestra exudados algodonosos en arcada temporal superior, exudados duros peripapilares, hemorragias puntiformes en el recorrido de las arcadas y cruces arteriovenosos en arcadas vasculares superior e inferior grado II y III. 

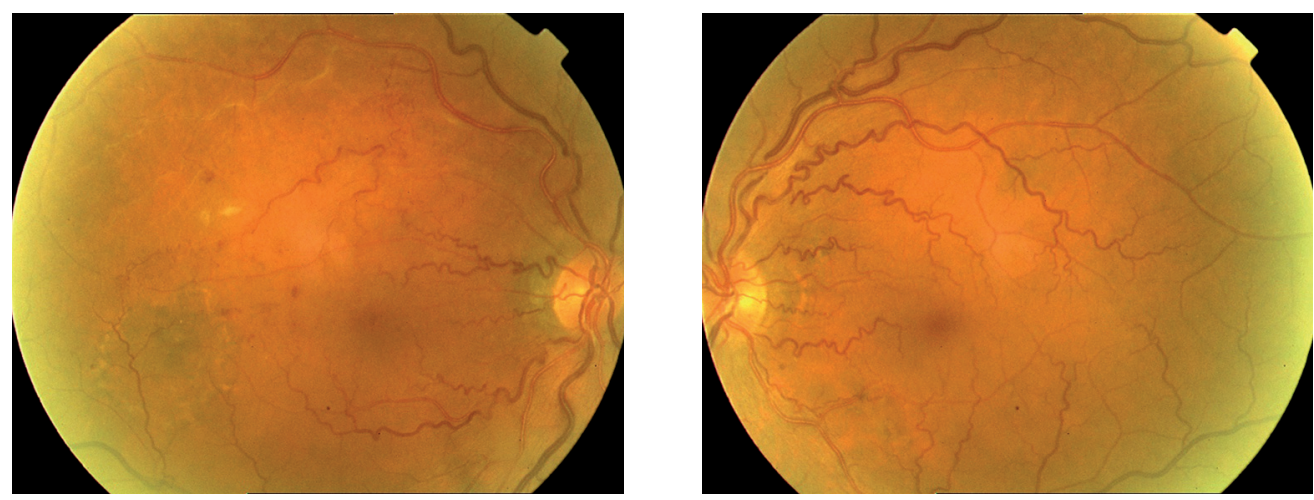

Figuras 2 y 3. Vasos retinianos tortuosos en forma de tirabuzón. Signo de Guist por tortuosidad de las vénulas perimaculares, dilatación venosa y cruces arteriovenosos grado III-IV en las arcadas temporales superiores.

- Grado II: compresión de la vena en el parénquima retiniano, con falta de la columna sanguínea antes y después del cruce.

- Grado III: compresión de la vena con dilatación del extremo distal venoso.

- Grado IV: exudados y hemorragias a nivel del cruce.

- Signo de Salus: valora la variación del trayecto venoso a nivel del cruce que va cambiando de un ángulo agudo normal para hacerse más perpendicular e incluso invertir la dirección de cruce. Hay tres grados:

- Grado I: ligera desviación del segmento venoso haciéndose algo mas perpendicular.

- Grado II: el segmento venoso se hace perpendicular adoptando una forma en bayoneta.

- Grado III: hay una inversión de la dirección venosa adoptando una forma en $\mathrm{z}$.

\section{Alteraciones del reflejo vascular}

El reflejo normal de los vasos retinianos muestra una estría luminosa en el centro del mismo y tiene su origen en la reflexión de la luz entre la interfase de la pared vascular y la sangre que contiene. Dicho reflejo es aproximadamente $1 / 3$ del calibre de la arteriola. Las modificaciones en esta característica que origina la HTA crónica son ${ }^{3}$ :

- Arteriola en hilo de cobre: aparece en las fases iniciales de la enfermedad. Hay un engrosamiento de la pared vascular por fibrosis, hialinización y a la vez estrechamiento de la luz vascular con lo que el reflejo vascular es más ancho y de aspecto metálico-cobrizo.

- Arteriola en hilo de plata: aparece en HTA de larga evolución en la que la fibrosis, la hialinización y la arterioesclerosis impiden la visualización de la columna sanguínea por lo que la pared del vaso refleja la mayor parte de la luz dando un reflejo amplio, brillante y blanquecino.

\section{Reducción del calibre de las arteriolas}

\section{Generalizado}

La disminución del calibre arteriolar es consecuencia de una vasoconstricción difusa. La relación normal entre arteriola y 
Tabla 1. Signos oftalmológicos de la retinopatía por HTA.

I. Síndrome vascular esclero-hipertensivo

1. Signos de cruce arteriovenoso

2. Disminución del calibre arteriolar

3. Alteraciones del reflejo vascular

4. Irregularidades del trayecto vascular

5. Aneurismas retinianos

II. Síndrome vascular hipertono-hipertensivo

1. Arterias y arteriolas estrechadas y rectas

2. Venulas tortuosas (signo de Guist)

3. Parénquima retiniano normal

III. Retinopatía esclero-hipertensiva
1. Trombosis venosa
2. Obstrucción arterial por fibrosis
3. Hemorragias aisladas e infartos
4. Atrofia retiniana y movilización de pigmento
5. Macroaneurismas

IV. Retinopatía hipertono-hipertesiva. Retinopatía de la hipertensión maligna

1. Vasoconstricción generalizada y envainamiento vascular

2. Edema retiniano difuso y estrella macular

3. Microaneurismas

4. Hemorragias retinianas superficiales y profundas

5. Exudados algodonosos y lipoideos

6. Desprendimientos serosos de retina por coroidopatia hipertensiva

7. Edema de papila óptica

vénula es de $3 / 4$ a $2 / 3$. Estos cambios son reversibles en las fases iniciales de la HTA al aplicar tratamiento médico antihipertensivo y no así en la HTA de larga evolución o HTA maligna. Los grados de este estrechamiento son ${ }^{3}$ :

- Grado I- Ligero: 2/3-1/2

- Grado II- Moderado: 1/2-1/3

- Grado III- Intenso: < 1/3

- Grado IV- Muy grave: arterias filiformes

\section{Focal}

Esta es debida a un vasoespasmo de la porción de la arteriola que todavía no ha sufrido esclerosis y en la que la capa muscular permanece indemne pudiendo adaptarse todavía a la HTA sistémica.

\section{Alteraciones del trayecto vascular}

Los vasos retinianos se pueden modificar por la HTA aumentando su tortuosidad, adoptando forma de tirabuzón, o bien adoptando una configuración más rectilínea y alargada. Así los principales signos a tener en cuenta son ${ }^{3}$ (Figs. 2 y 3 ):

- Signo de Bonnet: bifurcaciones arteriales en forma de letra Omega o ángulo recto.

- Signo de Guist: tortuosidad de las vénulas perimaculares (Fig. 2).

\section{Aneurismas retinianos}

Dentro de estas formaciones aparecen los microaneurismas y los macroaneurismas. Los microaneurismas se encuentran diseminados por toda la retina, pero más frecuentemente en las bifurcaciones arteriolares donde aparecen como una dilatación sacular generalmente trombosada. Para detectarse con claridad es necesaria la realización de una angiografía fluoresceínica.

Los macroaneurismas aparecen en las arteriolas en los tres primeros órdenes del árbol arterial. Suelen ser unilaterales en un 
90\% de los casos, aparecen en pacientes de edad avanzada (más de 65 años), sobre todo mujeres con hipertensión de larga evolución. Generalmente son asintomáticos aunque en algunos casos pueden sangrar, producir exudación con edema retiniano o exudados duros. Algunos de ellos se trombosan espontáneamente por lo que se resuelven solos y en los casos que producen edema retiniano con afectación macular es necesaria la fotocoagulación con láser Argón ${ }^{3,4}$.

\section{SIGNOS DE LA RETINOPATÍA HIPERTENSIVA}

\section{Hemorragias}

Las hemorragias a nivel retiniano se producen como consecuencia de las lesiones de las paredes vasculares y el aumento de la presión intravascular e hidrostática. En la HTA de larga evolución se producen lesiones en el endotelio vascular, depósitos de material fibroso y alteración de la estructura de la pared. Esto conlleva una pérdida de la capacidad de autorregulación, con dilatación de las arteriolas y pérdida de las uniones entre las células endoteliales y pericitos, con disrupción de la barrera hemato-retiniana, aumentando la permeabilidad de los vasos y extravasación de elementos formes sanguíneos a nivel intra-retiniano y superficial. En otros casos cuando la instauración de la HTA es de forma brusca no se producen fenómenos de adaptación por lo que se produce una rotura de todo el espesor de la pared del vaso dando lugar a hemorragias intraretinianas profundas ${ }^{5,6}$. Las hemorragias se clasifican en:

\section{Hemorragias retinianas}

- Hemorragias en astilla: se producen a nivel de la capa de fibras nerviosas de Henle adoptando una disposición lineal.

- Hemorragias puntiformes: se producen a nivel profundo en las capas nucleares interna y externa y capa plexiforme.

- Hemorragias en mancha: en la misma localización que las anteriores pero de mayor tamaño.

\section{Hemorragias conoideas}

Son profundas y de color rojo oscuro. En algunos casos son difíciles de observar al quedar ocultas por las alteraciones del epitelio pigmentario de retina o el edema retiniano difuso. Es importante señalar que cuando existen lesiones a nivel coroideo (coroidopatía hipertensiva) estamos ante un caso avanzado o maligno de la HTA que suele asociar insuficiencia renal ${ }^{5-7}$.

\section{Hemornagias prernetinianas o retrohialoideas}

Son densas, obscuras y se localizan por debajo de la membrana limitante interna o el espacio retrohialoideo adoptando forma curva inferior y rectilínea superior en forma de barca o medialuna.

\section{Hemornagia vítrea}

Ésta es una hemorragia retiniana o retrohialoidea que atraviesa la hialodes posterior e invade el vítreo pudiendo adoptar diferentes formas, bien un coágulo localizado o un hemovítreo difuso para finalmente organizarse formando un magma fibroso blanco amarillento que se dispone en la porción inferior del ojo.

\section{Exudados blandos o algodonosos}

Son manchas blanquecinas, difusas, de bordes mal definidas y de un diámetro similar a la papila a nivel de la capa de fibras nerviosas secundarias a infartos de esta capa por vasoconstricción arteriolar con el resultado de isquemia aguda y severa. Se localizan cerca de los capilares peripapilares, se asocian a microaneurismas y son un signo de entrada en la fase acelerada de la hipertensión o malignización de la misma. Su aparición también ha de alertar por el alto riesgo de fallo multiorgánico como renal y/o cardiaco ${ }^{7}$ (Fig.1).

\section{Exudados duros}

Son depósitos intrarretinianos de lípidos y proteínas blanquecinos, brillantes y bordes bien definidos. Aparecen como consecuencia de un aumento de permeabilidad vascular por isquemia prolongada o bien tras reabsorción de edemas o hemorragias retinianas como en el caso de las 
trombosis. Si estos depósitos se disponen en la región macular o perimacular en forma radial dan lugar a una estrella macular, signo de lesión avanzada con disminución de agudeza visual ${ }^{7}$ (Fig.4).

\section{Edema de papila}

Esta alteración representa un signo de mal pronóstico en los pacientes que no se logra controlar rápidamente la elevación tensional. Se observa una papila sobreelevada con bordes poco definidos y sin excavación fisiológica, con congestión venosa y en algunos casos hemorragias en astilla. Este edema puede ser el resultado de dos procesos fisiopatológicos $^{7,8}$ (Fig. 4):

- Hipertensión endocraneal: por encefalopatía hipertensiva y efecto mecánico.

- Isquemia de la papila: secundaria a la oclusión de las arteriolas a dicho nivel que producen hiperhidratación de las fibras del nervio óptico e interrupción del flujo axoplásmico que explicaría los exudados algodonosos y el consiguiente edema de papila. Debe considerarse

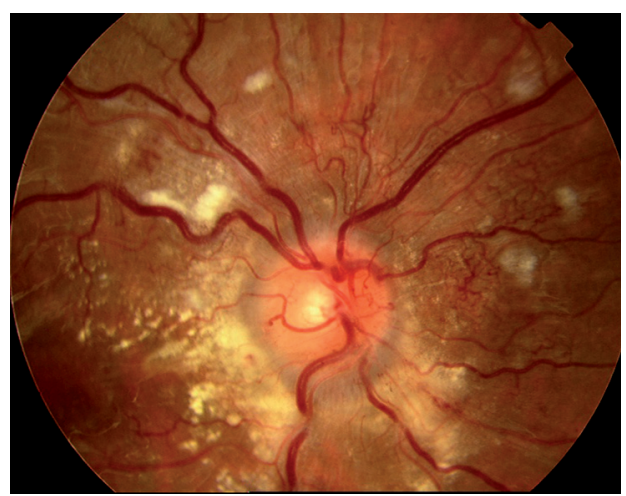

Figura 4. Edema de papila secundario a HTA maligna junto a exudados duros tortuosidad vascular, neovasos peripapilares y signos de cruce grado IV. en estos casos como una neuritis óptica isquémica anterior (NOIA).

Atendiendo a esta tipo de lesiones la clasificación de Keith-Wagener-Barker (Tabla 2) tiene importancia pronóstica:

- Grado I y II: pronóstico vital normal. No indica lesiones renales ni cardiovasculares.

- Grado III: esperanza de vida algo más de dos años. Indica daño a nivel de riñón, sistema nervioso central y corazón.

- Grado IV: muy mal pronóstico vital con esperanza de vida inferior a un año.

\section{COMPLICACIONES \\ OFTALMOLÓGICAS DE LA HIPERTENSION ARTERIAL}

\section{Atrofia de retina}

Se manifiesta como una reducción en el espesor retiniano por modificación de las células retinianas debido a anomalías en su funcionamiento, que conducen a un

Tabla 2. Clasificación de Keith-Wagener-Barker.

\begin{tabular}{l}
\hline Grado I \\
- Esclerosis arteriolar \\
- Arteriola en hilo de cobre o plata \\
- Constricció focal \\
- Poca o nula alteración de organos sistémicos \\
Grado II \\
- Constricción focal o difusa \\
- Cruces arteriovenoso \\
- Exudados duros \\
- Hemorragias puntiformes \\
- Trombosis venosas \\
- TA alta permanentemente sin daño sistémico \\
Grado III \\
- Edema retiniano y estrella macular \\
- Hemorragias retinianas \\
- Exudados algodonosos \\
- Cambios arteroscleróticos severos \\
- TA alta permanentemente con daño de órga- \\
nos sistémicos \\
Grado IV \\
- Lesiones anteriores más edema de papila \\
- Alteración grave en el SNC, riñón y otros \\
órganos \\
\hline
\end{tabular}


menor tamaño y número de las mismas secundaria a una esclerosis vascular intensa. Esto se manifiesta a nivel de fondo de ojo por áreas despigmentadas junto a otras de acúmulo de pigmento por la degradación del EPR ${ }^{9-11}$.

\section{Atrofia óptica}

Se produce por una degeneración isquémica de las fibras del nervio óptico secundario al edema de papila. Se caracteriza por una palidez del disco óptico y defectos irreversibles de la visión. Es de señalar que no existe una correlación exacta entre el grado de palidez y la agudeza visual. Atendiendo al aspecto del fondo de ojo hay tres tipos de atrofia óptica ${ }^{12,13}$ :

- Primaria: es la forma más frecuente y en ella la papila conserva unos límites netos y el disco está pálido.

- Secundaria: además de la palidez existe borrosidad de los márgenes papilares.

- Cavernosa: es similar a la atrofia glaucomatosa, con palidez, excavación central y desplazamiento nasal de los vasos.

\section{Embolia arterial}

En los pacientes con HTA maligna puede manifestarse como una embolia de arteria central de retina (OACR) o de alguna de las ramas arteriales, aunque es menos frecuente que la afectación venosa. Cursa con una pérdida indolora, irreversible y profunda de la agudeza visual que puede ser completa si afecta a la arteria central o sólo un sector del campo visual si afecta a una rama arterial. En la OACR se ve la clásica imagen de mancha rojo cereza en el fondo de ojo y en caso de ser una embolia de rama se aprecia edema localizado en la zona de la rama afecta. Otro signo que podemos ver en aproximadamente el 15-20\% de los casos es el émbolo causante de la obstrucción y la segmentación de la columna sanguínea ${ }^{9,10}$ (Fig. 5).

\section{Trombosis venosa}

Esta complicación es bastante frecuente en hipertensos de larga evolución y mal controlados pudiendo afectar a la vena central de retina (OVCR) y ser ésta a su vez

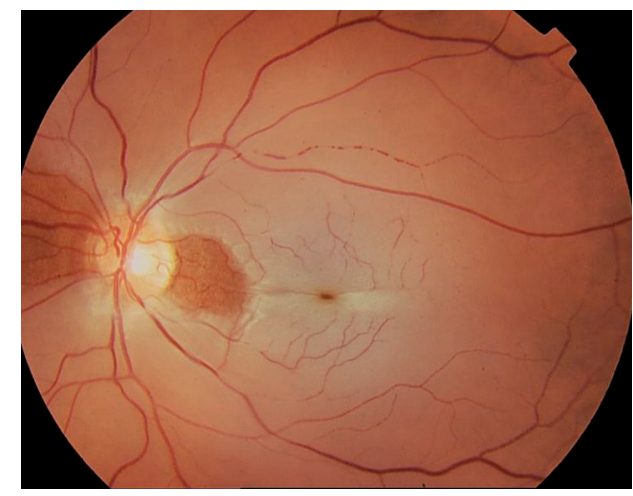

Figura 5. Embolia de arteria central de retina en la que se aprecia edema retiniano difuso, mancha rojo cereza, persistencia de la circulación cilioretiniana y segmentación de la columna sanguínea en la arteria temporal superior.

isquémica y no isquémica o afectar a una rama venosa (ORV) ${ }^{9,10}$ (Figs. 6 y 7).

\section{Oclusión de vena central de retina}

- OVCR isquémica: supone un tercio de los casos y cursa con una disminución severa de la agudeza visual (menor o igual a 1/10). En el fondo de ojo se aprecian abundantes hemorragias retinianas sobre todo en polo posterior, exudados algodonosos, dilatación y tortuosidad venosa con hiperemia $\mathrm{y}$ edema de papila. Como consecuencia de la isquemia tiene alto riesgo de complicaciones por neovascularización retiniana y de segmento anterior por lo que estos pacientes precisarán revisión mensual los primeros seis meses realizando fotocoagulación láser en caso de apreciarse neovasos retinianos o a nivel del iris.

- OVCR no isquémica: supone dos tercios de los casos y la disminución de visión es más leve (de 1/10 a 5/10). En el fondo de ojo se aprecian hemorragias dispersas en la periferia pero en menor cuantía que en la forma isquémica y menos edema de papila. Al ser menos grave la evolución es más favorable con desaparición de los signos 

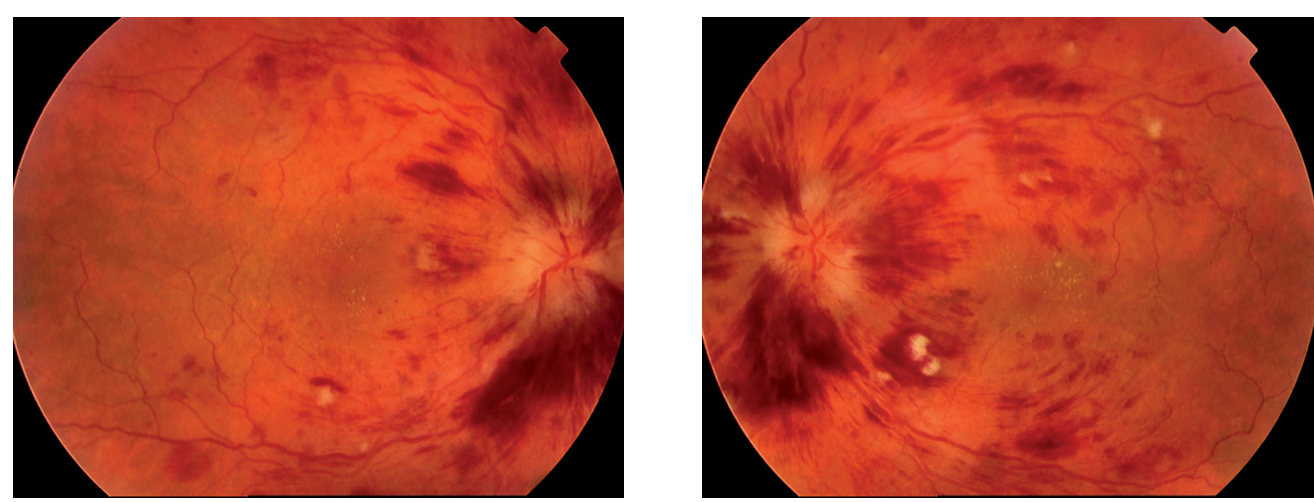

Figuras 6 y 7. Trombosis de la vena central retiniana bilateral no isquémica secundaria a hipertensión arterial crónica mal controlada. Abundante hemorragias en llama en el trayecto de las arcadas vasculares, exudados algodonosos y edema de papila bilateral con bordes poco nítidos y elevados.

agudos en 6-12 meses, aunque en un 10$15 \%$ de los casos puede evolucionar a la forma isquémica.

\section{Oclusión de rama venosa de retina}

La oclusión de rama venosa se suele originar a nivel de los cruces arteriovenosos por la compresión que sufre la vena en ese punto por la adventicia común engrosada, siendo la rama temporal superior la que se afecta con mayor frecuencia debido a un mayor número de cruces con la arteria. Clínicamente produce una disminución de visión o defecto del campo visual en caso de situarse la obstrucción cerca de la mácula. No obstante, en casos de afectación periférica puede pasar desapercibida para el paciente y no presentar síntomas. En el fondo de ojo se ve un área de lesión triangular de hemorragias en astilla y exudados algodonosos con el vértice en el lugar de la obstrucción para posteriormente y con el paso de los meses desparecer las hemorragias, aparecer exudados duros y finalmente quedar signos de oclusión antigua como envainamiento, cubrimiento vascular, colaterales, depósitos de cristales de colesterol y degeneración epitelial pigmentaria.

\section{Neovascularización}

Se origina secundaria a la isquemia retiniana y liberación de factores quimiotácti$\cos$ y angiogénicos que favorecen la proliferación vascular anómala por lo que son un indicativo de isquemia retiniana de larga evolución. Estos neovasos son serpenteantes, tortuosos, pierden su arquitectura normal y presentan una permeabilidad aumentada por lo que se acompañan de edema que en caso de afectar a la mácula produce importante pérdida visual. En algunos casos se observa cómo pueden penetrar en la cavidad vítrea, presentando un alto riesgo de sangrado ante traumatismos mínimos ${ }^{11}$.

\section{Desprendimiento de retina}

$\mathrm{Al}$ igual que en las embolias, es más frecuente en los casos de HTA maligna (Figs. 8 y 9), dando lugar a oclusiones e infartos agudos de la coriocapilar produciendo una alteración del epitelio pigmentario suprayacente de la retina alterando la barrera hematorretiniana y posteriormente un desprendimiento seroso de la retina neurosensorial que puede ser focal o difuso. Estos desprendimientos suelen ser bilaterales y focales, y si se extienden a la zona 

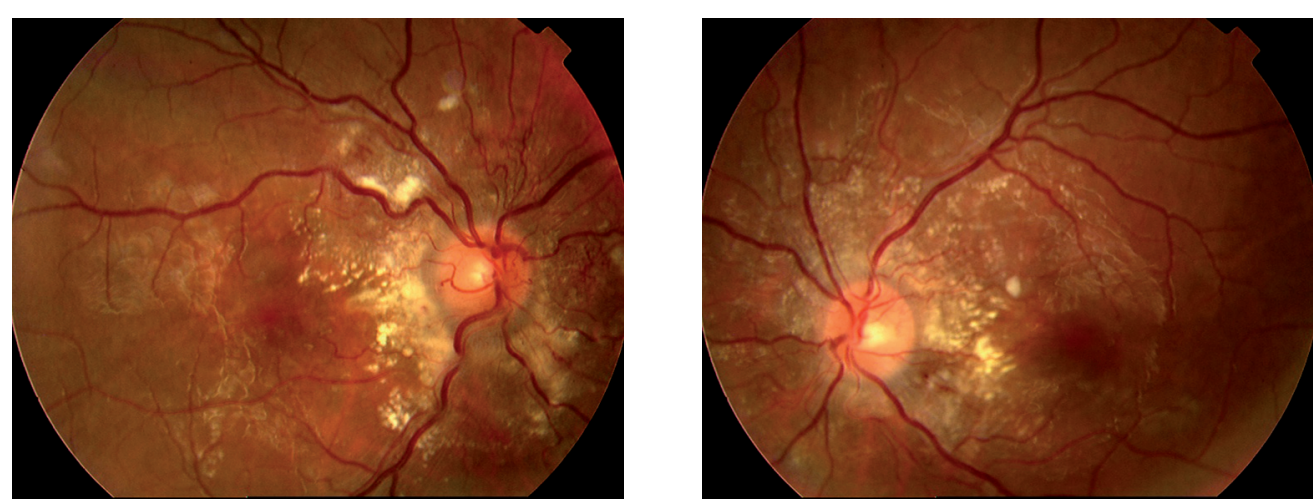

Figuras 8 y 9. Fondo de ojo en una hipertensión arterial maligna no controlada con exudados algodonosos, exudados duros, edema retiniano, neovasos tortuosos en tirabuzón peripapilares, alteraciones del trayecto vascular y edema de papila.

inferior se pueden hacer bullosos manifestándose como una área blanco-grisácea elevada. Posteriormente cuando la circulación se restaura la retina desprendida puede aplicarse apareciendo manchas que son zonas atróficas amarillentas junto a acúmulos focales de pigmento. Dentro de estas manchas encontramos las manchas de Elschning (redondeadas con un centro pigmentado y un halo atrófico blanco-amarillento) y las manchas de Siegrist (estrías en la periferia retiniana) $)^{14}$.

\section{Otras complicaciones}

Otras complicaciones que pueden aparecer son:

- Progresión de la retinopatía diabética.

- Hemorragia vítrea.

- Infartos coroideos isquémicos (manchas de Elschnig).

\section{BIBLIOGRAFIA}

1. SÁnchez Salorio M, Díaz-Llopis M, Benítez del CAstillo JM, RodríGuez Ares MT. Manifestaciones oftalmológicas de las enfermedades generales. LXXVII Ponencia Oficial de la Sociedad Española de Oftalmología 2001.
2. Tso MO, Jampol LM. Pathophysiology of hypertensive retinopathy. Ophthalmology 1982; 89: 1132-1145.

3. HAYREH SS. Classification of hypertensive fundus changes and their order of appearence. Ophthalmologica 1989; 198: 247-260.

4. WALSH J. Hypertensive retinopathy. Description, classification and prognosis. Ophthalmology 1982; 89: 1127-1131.

5. Hurcomb PG, Wolffsohn JS, Napper GA. Ocular signs of systemic hypertension: a review. Ophthalmic Physiol Opt 2001; 21: 430-440.

6. HAYREH SS, SERVAIS GE, VIRDI PS. Hypertensive retinopathy. Introduction. Ophthalmologica 1989; 198: 173-177.

7. Tso M, Abrams G, JAMPol L. Hypertensive retinopathy, choroidopathy, and optic neuropathy. A clinical and pathophysiological approach to classification. En: Retinal and choroidal manifestation of systemic disesase. Singermann L, Jampol L. Williams \& Wilkins. Baltimore, 1991; 79.

8. Dodson PM, LiP GY, EAMES SM, GIBSON JM, BEEvers DG. Hypertensive retinopathy: a review of existing classification systems and a suggestion for a simplified grading system. J Hum Hypertens 1996; 10: 93-98.

9. Hubbard LD, Brothers RJ, King WN, ClegG LX, KLEIN R, CoOper LS et al. Methods for 
evaluation of retinal microvascular abnormalities associated with hypertension/sclerosis in the Atherosclerosis Risk in Communities Study. Ophthalmology 1999; 106: 2269-2280.

10. Sánchez Salorio M, Pita Salorio D. Atlas de retinopatías vasculares. Sandoz. Barcelona. 1972; 2: 13-38.

11. HaYreh SS, SERVAIS GE, VIRDI PS. Fundus lesions in malignant hypertension. IV. Focal intraretinal periarteriolar transudates. Ophthalmology 1986; 93: 60-73.
12. Hayreh SS, SERVAIS GE, VIRdi PS. Fundus lesions in malignant hypertension. V: Hypertensive optic neuropathy. Ophthalmology 1986; 93: 74-78.

13. Hayreh SS, Servais GE, VIRdi PS. Fundus lesions in malignant hypertension. VI: Hypertensive choroidopathy. Ophthalmology 1986; 93: 1383-1400.

14. De Venecia G, Jampol LM. The eye in accelerated hypertensión. II. Localized serous detachments of the retina in patients. Arch Ophthalmol 1984; 102: 68-73. 